Oral presentation

\title{
Engineering a synthetic molecular oscillator based on the Lotka-Volterra dynamic
}

Christin Sander*1, Farah Vohra1, John Chattaway², Tom Hinson ${ }^{3}$, John Sy1, Jiongjun Bai ${ }^{4}$, Jonathan Wells' ${ }^{2}$, Deepti Aswani², Vincent Rouilly1, ChuehLoo Poh ${ }^{1}$, Matthieu Bultelle ${ }^{1}$, Kirsten Jensen ${ }^{3}$, David Mann ${ }^{3}$, Paul Freemont ${ }^{3}$ and Richard Kitney*1

Address: ${ }^{1}$ Department of Bioengineering, Imperial College, London, SW7 2AZ, UK, ${ }^{2}$ Department of Biology, Imperial College, London, SW7 2AZ, UK, ${ }^{3}$ Department of Biochemistry, Imperial College, London, SW7 2AZ, UK and ${ }^{4}$ Department of Electrical Engineering, Imperial College, London, SW7 2AZ, UK

Email: Richard Kitney* - r.kitney@imperial.ac.uk

* Corresponding authors

from BioSysBio 2007: Systems Biology, Bioinformatics and Synthetic Biology Manchester, UK. II-13 January 2007

Published: 8 May 2007

BMC Systems Biology 2007, I (SuppI I):S9 doi:I0.II86/I752-0509-I-SI-S9

This abstract is available from: http://www.biomedcentral.com/I752-0509/I?issue=SI

(C) 2007 Sander et al; licensee BioMed Central Ltd.

\section{Background}

Oscillators are a fundamental building block in many engineering fields, as they provide the basis for counting, timing and synchronisation. Oscillators are found in many everyday devices such as clocks, computers or radios. Similarly, oscillations are an essential part of biological systems - providing the basis for, for example, rhythmic patterns and regulatory networks. The ability to build a stable, controllable biological oscillator would be a major step towards reliable synthetic biology based circuits. Elowitz et al. were part of the first ones to try to build an oscillator. Their oscillator was based on genetic network at the single cell level. However, due to the stochastic behaviour inherent at the gene expression level, the oscillations were not stable or persistent. In this paper, we present an original oscillator design produced during the iGEM-2006 competition at Imperial College. The project addressed stability and reliability issues by defining an oscillator at the population level and by applying strict engineering rules to the system development process.

\section{Methods}

This iGEM project combined genetic engineering methods with a clearly defined development cycle, copied from the traditional engineering approach - a cycle of specification, design, modelling, testing and implementation with biological processes to produce a synthetic biological oscillator. The overall specification aim was to build a biological oscillator in E. Coli based on the Lotka-Volterra population dynamics. This approach was used to design a synthetic quorum sensing \& quenching mechanism, made of BioBricks. In order to satisfy the requirements for the Lotka Volterra predator-prey dynamics BioBricks were constructed to show properties such as exponential growth of a prey molecule (Acyl Homoserine Lactone). In order to reduce the complexity of the overall assembly, test constructs were built to characterise the different system components. Because the design comprises BioBricks in a two population system, the parameters of the oscillations can be controlled to achieve synchronised oscillations. Furthermore, since the system uses population wide oscillations, it enables other devices to synchronise the oscillator. It is important to determine the component properties and their impact on the overall system. Hence, the modelling of the pure and modified Lotka-Volterra dynamics, along with the test constructs, were carried out. Because BioBricks were used in the design, the implementation was carried out with standard assembly. At the test- 


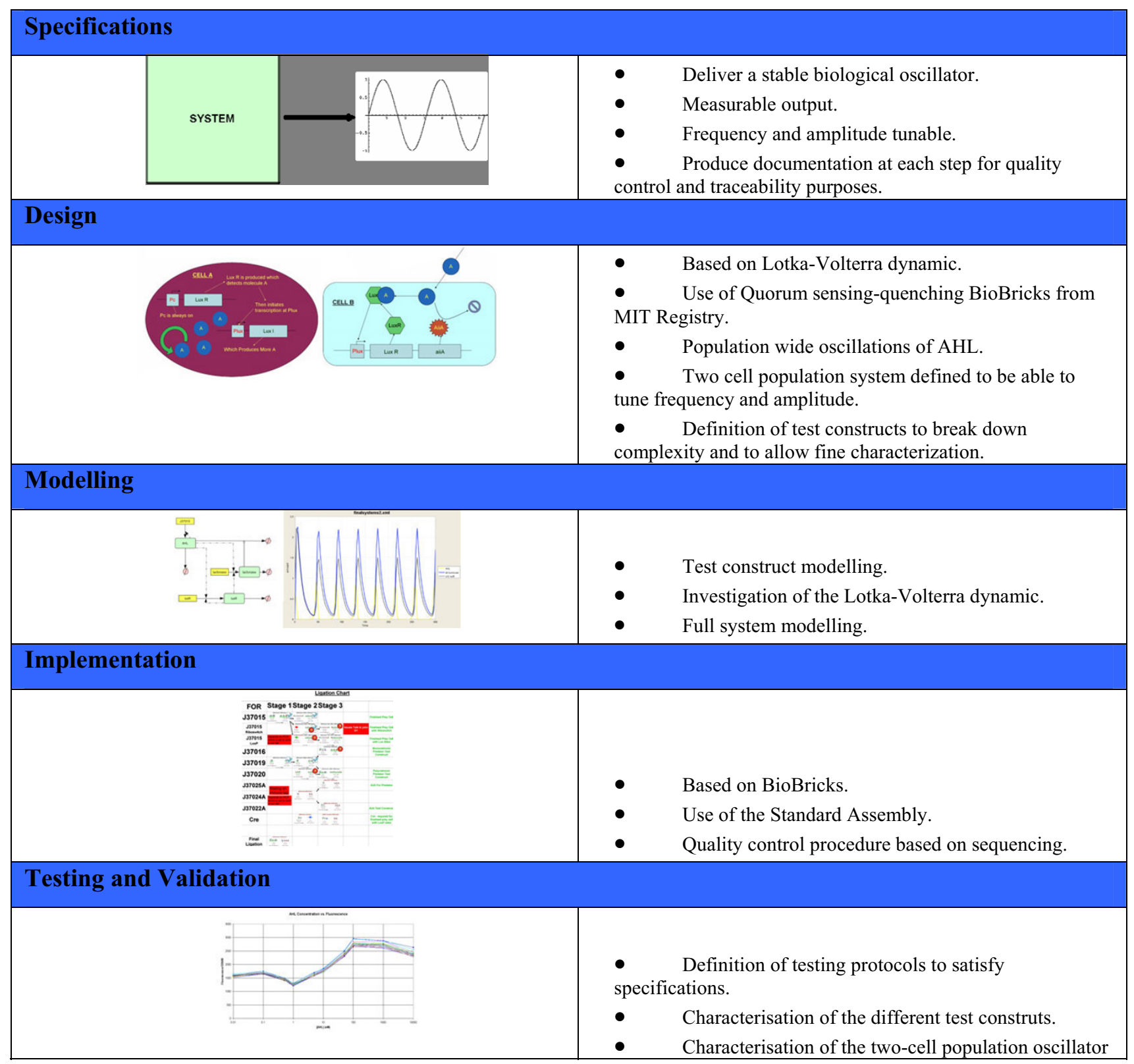

Figure I

Summary of our methodology.

ing stage, the test constructs were characterised. Consequently, the properties and constraints on the full system could be extracted. (See a summary of the method in Figure 1)

\section{Results}

BioBricks composing the test constructs and the final oscillator were engineered and a number of important results were extracted from the modelling and testing stages: i) The test constructs/BioBricks representing the different parts of the oscillator were characterised from testing in E. Coli (DH5 $\alpha)$. ii) The model of the test constructs, which included experimental data, predicted stable oscillations as output of the full system. iii) The influence of specific system parameters on the frequency, amplitude and stability of the oscillations was investigated with the Lotka-Volterra model. 


\section{Conclusion}

In the project, fundamental units of the system were fabricated and characterised. These have been added to the Registry of Standard Biological Parts. The modelling exercise was highly successful and we fully expect to complete a stable synthetic biology based oscillator to be completed in the near future. For full details visit our iGEM website http://openwetware.org/wiki/IGEM:IMPERIAL/2006.

Publish with Bio Med Central and every scientist can read your work free of charge

"BioMed Central will be the most significant development for disseminating the results of biomedical research in our lifetime. " Sir Paul Nurse, Cancer Research UK

Your research papers will be:

- available free of charge to the entire biomedical community

- peer reviewed and published immediately upon acceptance

- cited in PubMed and archived on PubMed Central

- yours - you keep the copyright

Submit your manuscript here:

http://www.biomedcentral.com/info/publishing_adv.asp 